\title{
Layer-by-Layer Nanoassembly of Copper Indium Gallium Selenium Nanoparticle Films for Solar Cell Applications
}

\author{
A. Hemati, S. Shrestha, M. Agarwal, and K. Varahramyan \\ Integrated Nanosystems Development Institute (INDI), Indiana University-Purdue University Indianapolis (IUPUI), \\ Indianapolis, IN 46202, USA \\ Correspondence should be addressed to S. Shrestha, sudshres@iupui.edu
}

Received 5 June 2012; Accepted 4 September 2012

Academic Editor: Gaurav Mago

Copyright ( $\odot 2012$ A. Hemati et al. This is an open access article distributed under the Creative Commons Attribution License, which permits unrestricted use, distribution, and reproduction in any medium, provided the original work is properly cited.

Thin films of CIGS nanoparticles interdigited with polymers have been fabricated through a cost-effective nonvacuum film deposition process called layer-by-layer (LbL) nanoassembly. CIGS nanoparticles synthesized by heating copper chloride, indium chloride, gallium chloride, and selenium in oleylamine were dispersed in water, and desired surface charges were obtained through $\mathrm{pH}$ regulation and by coating the particles with polystyrene sulfonate (PSS). Raising the $\mathrm{pH}$ of the nanoparticle dispersion reduced the zeta-potential from $+61 \mathrm{mV}$ at $\mathrm{pH} 7$ to $-51 \mathrm{mV}$ at $\mathrm{pH}$ 10.5. Coating the CIGS nanoparticles with PSS (CIGS-PSS) produced a stable dispersion in water with $-56.9 \mathrm{mV}$ zeta-potential. Thin films of oppositely charged CIGS nanoparticles (CIGS/CIGS), CIGS nanoparticles and PSS (CIGS/PSS), and PSS-coated CIGS nanoparticles and polyethylenimine (CIGS-PSS/PEI) were constructed through the LbL nanoassembly. Film thickness and resistivity of each bilayer of the films were measured, and photoelectric properties of the films were studied for solar cell applications. Solar cell devices fabricated with a $219 \mathrm{~nm}$ CIGS film, when illuminated by $50 \mathrm{~W}$ light-source, produced $0.7 \mathrm{~V}$ open circuit voltage and $0.3 \mathrm{~mA} / \mathrm{cm}^{2}$ short circuit current density.

\section{Introduction}

Layer-by-layer (LbL) nanoassembly is considered as one of the most precise, readily scalable, and cost-effective techniques for the thin film deposition of polymers and nanoparticles $[1,2] . \mathrm{Cu}(\mathrm{InGa}) \mathrm{Se}_{2}$ (CIGS) nanoparticles have been widely considered in thin film solar cells due to their high theoretical efficiency and tunable band gap. Typically, CIGS solar cells are fabricated using a coevaporation technique requiring a vacuum environment, and therefore resulting in higher cost $[3,4]$. Manufacturing costs for CIGS-based solar cells must be reduced to make economically viable. Many researchers have focused on lowering fabrication costs through alternate, nonvacuum deposition processes, such as blade coating and spray deposition technics $[5,6]$. However, these methods do not provide sufficient and accurate film thickness and produce undesirable rough surfaces [7]. LbL processes can be used to fabricate precisely tailored thin film on various types of substrates, both rigid and flexible. In LbL nanoassembly, positive and negatively charged species are sequentially deposited on a substrate via alternate dipping
[8]. LbL nanoassembly does not require sophisticated lab facilities or precise process control, thus yields a cost-effective thin film manufacturing process.

In this study, LbL nanoassembly is utilized to deposit controlled thin films of oppositely charged CIGS nanoparticles and polymers. Film deposition and electrical characterization results are presented and discussed. Novel aspects of this research include the functionalization of CIGS nanoparticles to create stable aqueous dispersions with desired surface charges, fabrication of thin CIGS film using LbL nanoassembly, and characterization and utilization of the films for solar cell application.

\section{Experimental}

2.1. CIGS Nanoparticle Synthesis. CIGS nanoparticles were synthesized using copper chloride $(\mathrm{CuCl})$, indium chloride $\left(\mathrm{InCl}_{3}\right)$, gallium chloride $\left(\mathrm{GaCl}_{3}\right)$, and selenium $(\mathrm{Se})$ in oleylamine (OLA) solvent. OLA (70\%) was heated at $200^{\circ} \mathrm{C}$ for 2 hours under nitrogen. Then, in nitrogen-filled glove box, $10 \mathrm{ml}$ of heated OLA was added to Se and heated at $250^{\circ} \mathrm{C}$ 
for 1 hour while being stirred. At the same time OLA was added into two different flasks containing $\mathrm{InCl}_{3}$ and $\mathrm{CuCl}$, each heated at $250^{\circ} \mathrm{C}$ for $30 \mathrm{~min}$ while being stirred. All three flasks were then mixed and $\mathrm{GaCl}_{3}$ was added. This reaction mixture was heated at $250^{\circ} \mathrm{C}$ for $75 \mathrm{~min}$ while being stirred [9-11]. The resulting nanoparticles were washed through suspension in chloroform and precipitation by ethanol. The size of the synthesized nanoparticles was analyzed using Malvern Nanosizer, size and zeta-potential measurement instrument, and atomic force microcopy (AFM), and material composition was studied through electron dispersive spectroscopy (EDS).

CIGS nanoparticles were cleaned through sonicating in ethanol for several hours and precipitating by centrifuging at a high speed. The resulting nanoparticles were dispersed in water. Zeta-potential $(\zeta)$ of the nanoparticles dispersed in water was controlled through $\mathrm{pH}$ regulation by slowly adding $0.1 \mathrm{~g} / \mathrm{ml} \mathrm{NaOH}$ solution to the CIGS dispersion. In another process, CIGS nanoparticles were coated with polystyrene sulfonate (PSS) to allow the uniform dispersion in water (CIGS-PSS).

2.2. Film Fabrications and Characterization. LbL nanoassembly is based on the sequential deposition of oppositely charged polyelectrolytes or nanoparticles. LbL films can be deposited on a wide variety of rigid and flexible substrates of different shapes and sizes [8]. LbL nanoassembly of oppositely charged CIGS nanoparticles (CIGS/CIGS), CIGS nanoparticles and PSS (CIGS/PSS), and PSS-coated CIGS nanoparticles and polyethylenimine (CIGS-PSS/PEI) was conducted.

To study the film development process, LbL nanoassembly was performed on a quartz crystal microbalance (QCM) [12]. The aqueous dispersion of CIGS nanoparticles and polymers was used for the deposition through alternately dipping the substrate in oppositely charged materials. The samples were washed in deionized water following each deposition. Separate LbL films of CIGS dispersed in water at $\mathrm{pH} 7(\zeta=+61 \mathrm{mV})$ and at $\mathrm{pH} 10.5(\zeta=-51 \mathrm{mV})$ (CIGS/CIGS film), CIGS at $\mathrm{pH} 7$ and PSS $(\zeta=-58 \mathrm{mV})$ (CIGS/PSS film), CIGS at $\mathrm{pH} 10.5$ and PEI $(\zeta=+16 \mathrm{mV})$ (CIGS/PEI film), and CIGS coated with PSS (CIGS-PSS, $\zeta=$ $-56.9 \mathrm{mV}$ ) and PEI (CIGS-PSS/PEI film) were deposited on QCM.

To measure thickness, films were scratched using micromanipulator probe and were analyzed using atomic force microscopy (AFM). Confocal scanning microscopy was used to take the images of the first and last layer of a CIGSPSS/PEI film composed of 25 bilayers. The bottom layer was lablled using Invitrogen's $20 \mathrm{~nm}$ fluorospheres in nile red (red-colored fluorescent dye) and the top layer was lablled using fluorescein isothiocyanate (FITC) (green-colored fluoerescent dye). To study the resisitivity and the photoelectric properties CIGS/CIGS, CIGS/PSS, and CIGS-PSS/PEI LbL films were deposited across a $200 \mu \mathrm{m} \times 58 \mathrm{~cm}$ channel on indium-tin-oxide- (ITO-) coated glass substrate. The current-voltage measurements of the films were conducted using Keithly Semiconductor Characterization instrument.

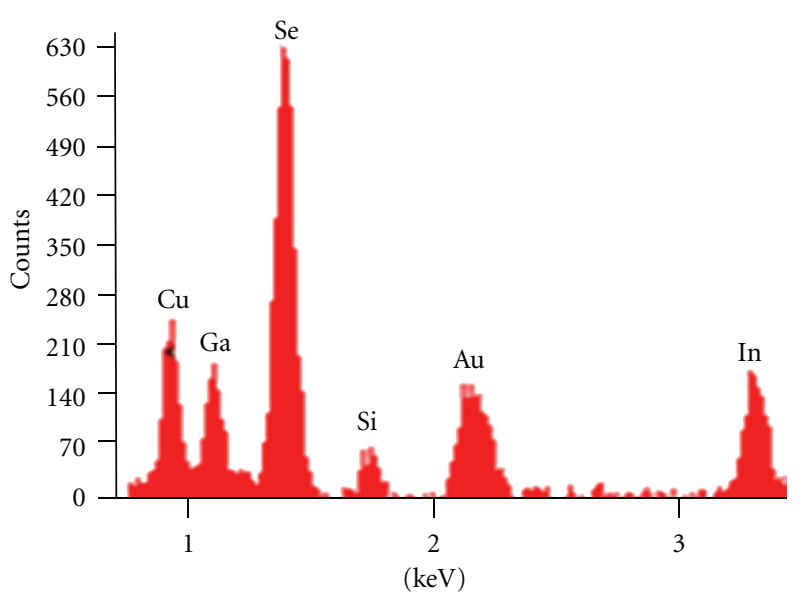

FIGURE 1: EDS results showing the material composition of CIGS nanoparticles placed on a gold-coated silicon substrate.

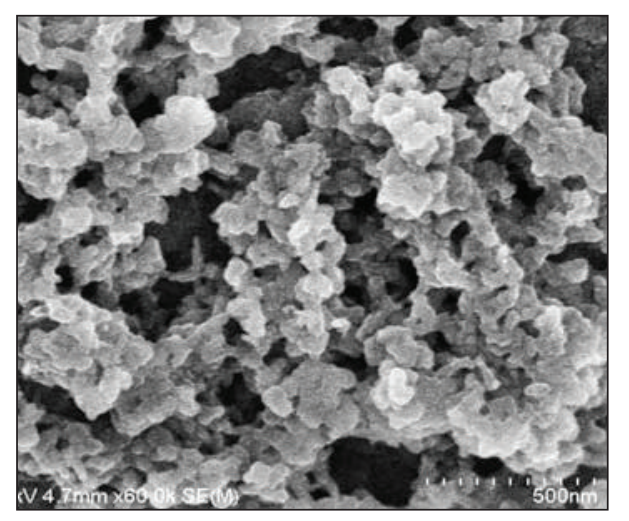

FIGURE 2: SEM image of CIGS nanoparticles in water.

The optical absorbance of a CIGS-PSS/PEI film deposited on a glass substrate was measured with ultraviolet-visible-near infrared (UV-Vis-NIR) spectroscopy.

2.3. Solar Cell Fabrications and Testing. A solar cell device consisting of CIGS-PSS/PEI absorbing layer, cadmium sulfide $(\mathrm{CdS})$ n-type layer, intrinsic zinc oxide $\left(\mathrm{ZnO}_{2}(\mathrm{i})\right)$ buffer layer, and aluminum-doped zinc oxide $\left(\mathrm{ZnO}_{2}(\mathrm{n})\right)$ layer was fabricated and tested. A $219 \mathrm{~nm}$ thick CIGS-PSS/PEI LbL film was deposited on an ITO-coated glass substrate. A thin cadmium sulfide film was deposited on the CIGSPSS/PEI through chemical bath deposition. Intrinsic zinc oxide $\mathrm{ZnO}_{2}(\mathrm{i})$ and $\mathrm{ZnO}_{2}(\mathrm{n})$ films were deposited by spincoating of the nanopowders dispersed in isopropyl alcohol. The J/V characteristics of the solar cell were measured with the Keithly Semiconductor Characterization instrument.

\section{Results and Discussion}

3.1. CIGS Nanoparticles Characterization. The EDS results showing the material composition of the synthesized CIGS nanoparticles are presented in Figure 1. The gold and silicon peaks are attributed to the gold-coated silicon substrate used. 


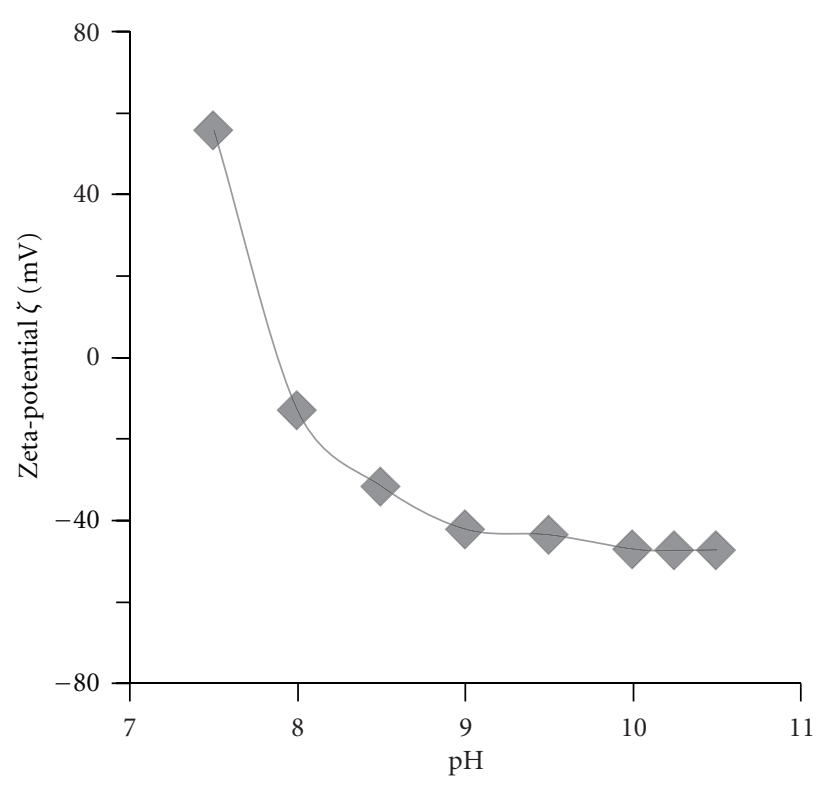

(a)

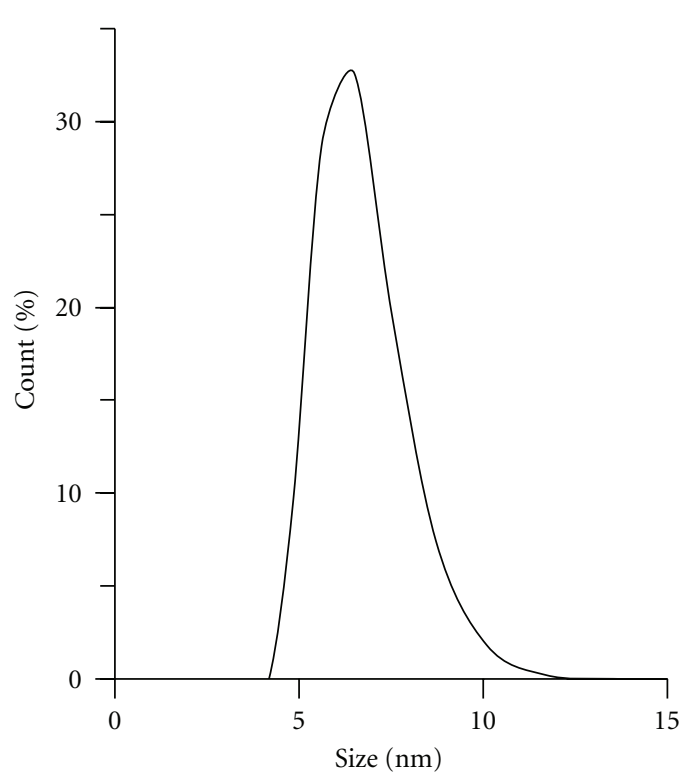

(b)

FIGURE 3: pH-dependent zeta-potential $(\zeta)$ of the CIGS nanoparticles dispersed in water. (b) CIGS-PSS nanoparticles dispersed in water.

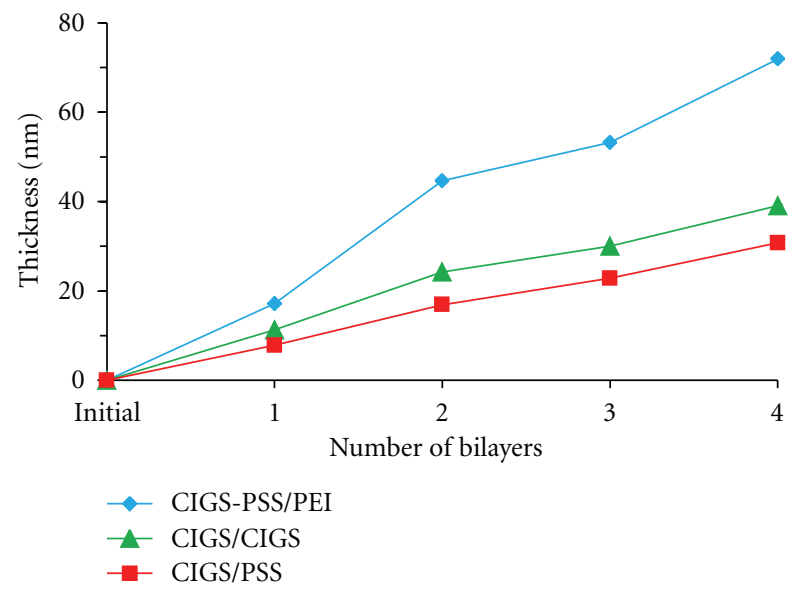

FIGURE 4: QCM results showing the film deposition of CIGSPSS/PEI, CIGS/CIGS, and CIGS/PSS.

A scanning electron microscopy (SEM) image of the CIGS nanoparticles is shown in Figure 2. The sample for the image was prepared by drying a drop of CIGS dispersion on a gold-coated silicon substrate. It is observed that while the individual particle sizes are in $10 \mathrm{~nm}$ range, it forms an aggregation when dried. Zeta-potential $(\zeta)$ of the CIGS nanoparticles dispersed in water was measured to be $+61 \mathrm{mV}$. The $\mathrm{pH}$ of the solution was raised by slowly adding $0.1 \mathrm{~g} / \mathrm{ml}$ $\mathrm{NaOH}$ solution to the CIGS dispersion. The change in the $\zeta$ of the nanoparticles dispersed in water with increasing $\mathrm{pH}$ is shown in Figure 3(a). It is observed that the $\zeta$ changes from $+61 \mathrm{mV}$ at $\mathrm{pH} 7$ to $-51 \mathrm{mV}$ at $\mathrm{pH}$ 10.5. As PSS was slowly added to the CIGS dispersed in water at $\mathrm{pH} 7$, the $\zeta$ of the nanoparticles slowly decreased from

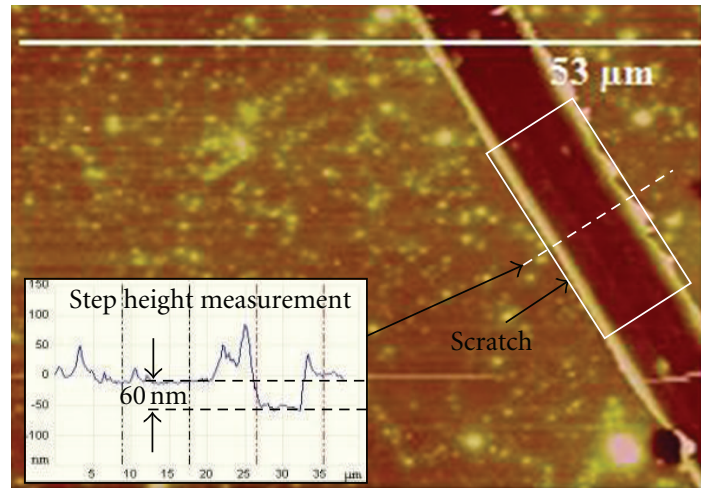

FIGURE 5: AFM image showing the top surface of a CIGS-PSS/PEI film. The inset shows the step height measurement of the scratch.

$+61 \mathrm{mV}$ to $-56.9 \mathrm{mV}$. The size distribution of the CIGS-PSS nanoparticles in water after separating bigger particles by centrifuging the dispersion at $15000 \mathrm{rpm}$ for 15 minutes is shown in Figure 3(b). The size distribution of the particle is observed to spread from 5-10 nm.

3.2. LbL Film Fabrications and Characterization. LbL film development processes of CIGS/CIGS, CIGS CIGS/PSS, CIGS/PEI, and CIGS-PSS/PEI films measured on a QCM are shown in Figure 4. The measured thicknesses after the addition of each bilayer of the films are presented. The thicknesses of 4 bilayers of CIGS/CIGS, CIGS CIGS/PSS, CIGS/PEI, and CIGS-PSS/PEI films were measured to be $31 \mathrm{~nm}, 38 \mathrm{~nm}$, and $70 \mathrm{~nm}$, respectively. The CIGS-PSS/PEI film shows the best film development among the three despite having good zeta-potentials of the CIGS in water at pH $7(+61 \mathrm{mV})$ and $\mathrm{pH} 10.5(-51 \mathrm{mV})$. The CIGS coated 


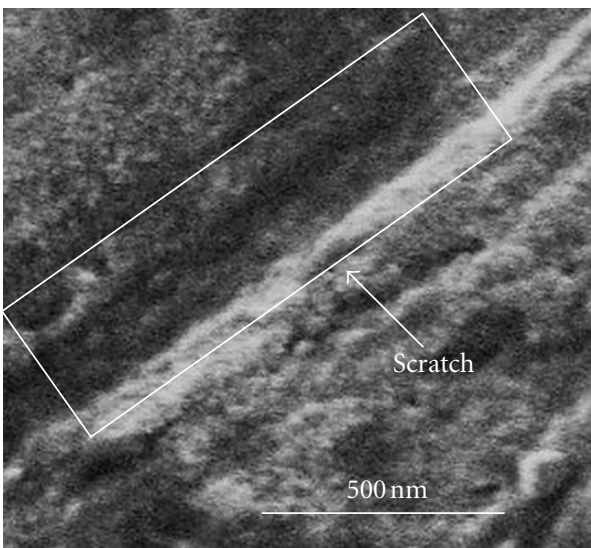

(a)

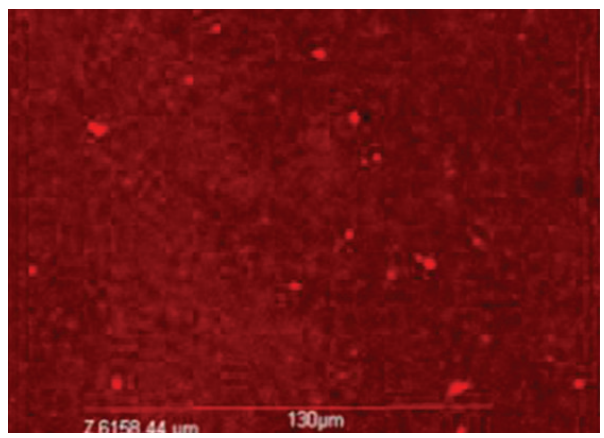

(c)

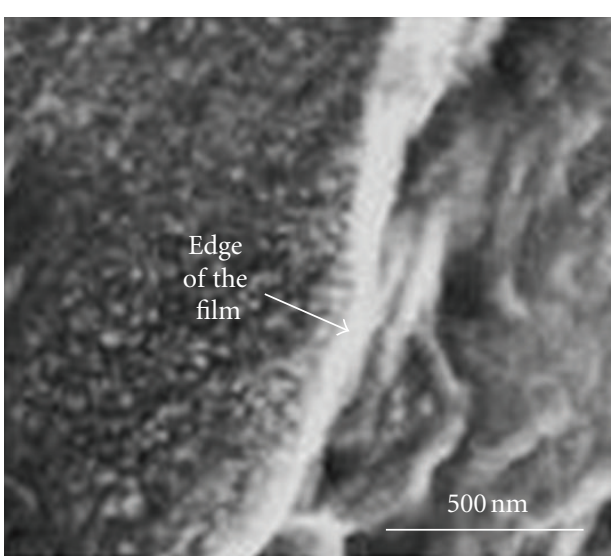

(b)

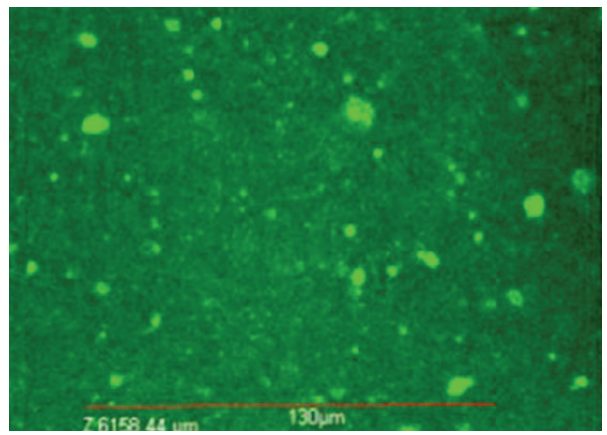

(d)

FIGURE 6: SEM images of a 6 bilayer LbL nanoassembled CIGS film. (a) Top surface. (b) The edge of the film. Confocal images of the (c) bottom and (d) top layer of a CIGS-PSS/PEI film.

with PSS shows a better adsorption to the surface than bare particles. It is attributed to a better attachment between the two polymers PSS and PEI, compared to the attachments between CIGS and PSS or CIGS and CIGS. Moreover, the comparable thicknesses of CIGS/CIGS and CIGS/PSS films further show that the attachment of CIGS to PSS is better than CIGS to CIGS.

AFM image showing the top surface of a CIGS-PSS/PEI film composed of 6 bilayers is presented in Figure 5. A scratch made using micromanipulator probe is also seen in the figure. The depth of the scratch was measured to be $60 \mathrm{~nm}$ using step height measurement tool within AFM. The depth of the scratch measured is smaller than the film thickness given by the QCM result. It shows that the scratch does not completely penetrate through the film. SEM images of a 6 bilayer CIGS-PSS/PEI film are shown in Figure 6. Figure 6(a) shows the top surface of the film illustrating a scratch made by the micromanipulator probe. Figure 6(b) shows the edge of the film. The SEM images confirm the solid film formed of the nanoparticles. Confocal scanning microscopy images of the first (bottom) and last (top) layers of a CIGS-PSS/PEI film taken with the help of fluorescent dyes are shown in Figures 6(c) and 6(d), respectively. The distinct colors of the bottom and the top surfaces of the film show that the layers are separated.

The resistivity of a $200 \mu \mathrm{m} \times 58 \mathrm{~cm}$ channel on an ITO-coated glass was $47 \mathrm{M} \Omega \cdot \mathrm{m}$. The decrease in measured

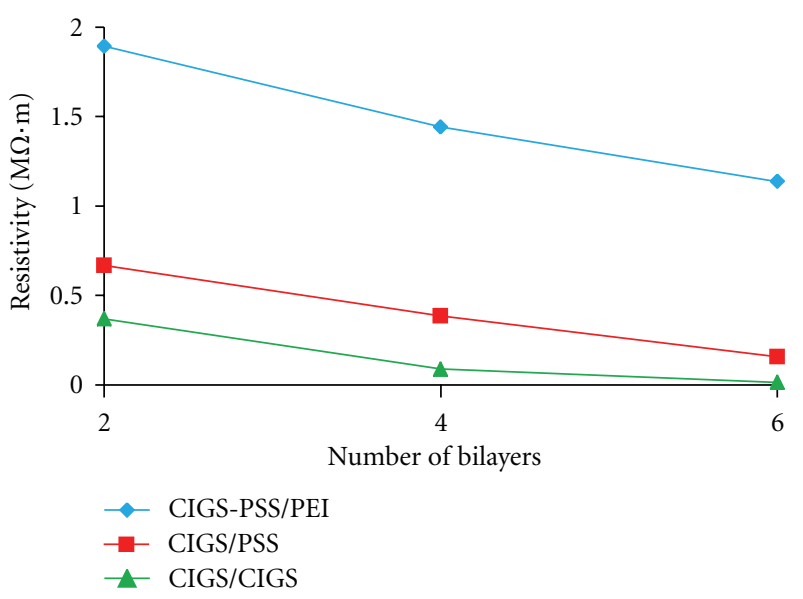

FIGURE 7: Resistivity versus number of bilayers of the deposited film of CIGS/CIGS, CIGS-PSS/PEI, and CIGS/PSS.

resistivity of the films with the deposition of every 2 bilayers of the films is shown in Figure 7. The resistivity for 6 bilayers of CIGS-PSS/PEI, CIGS/PSS, and CIGS/CIGS films was measured to be $1.13 \mathrm{M} \Omega \cdot \mathrm{m}, 0.15 \mathrm{M} \Omega \cdot \mathrm{m}$, and $0.01 \mathrm{M} \Omega \cdot \mathrm{m}$, respectively. The CIGS-PSS/PEI film has a higher resistivity due to the introduced PSS and PEI polymers. The CIGS/CIGS film has the lowest resistivity as expected. 


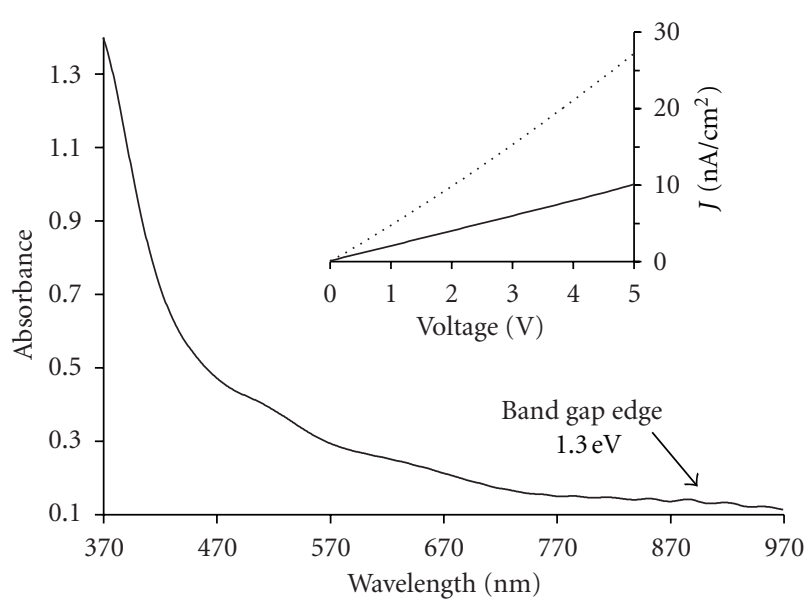

FIgURE 8: Absorbance spectrum of a CIGS-PSS/PEI film. The inset shows the current-voltage $(\mathrm{J} / \mathrm{V})$ characteristics of 6 bilayers of CIGS-PSS/PEI film in the dark (solid line) and under illumination (dotted line).

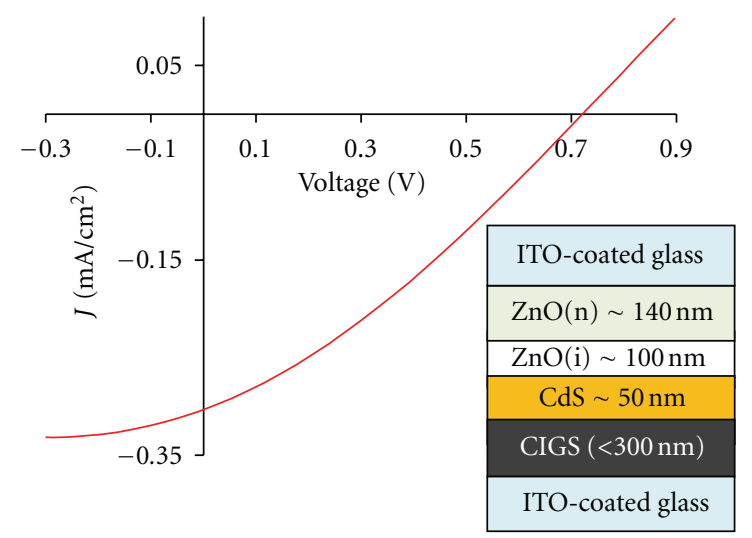

FIGURE 9: J/V characteristic of a solar cell device consisting of CIGSPSS/PEI, CdS, $\mathrm{ZnO}(\mathrm{n})$, and $\mathrm{ZnO}$ (i) films with ITO as both back and front contacts, when illuminated by $50 \mathrm{~W}$ light source. The inset shows the schematic of the device structure.

The optical absorbance of a CIGS-PSS/PEI film deposited on a glass substrate is shown in Figure 8. The results show that the absorbance spectrum edge of the film is near $920 \mathrm{~nm}$ wavelength ( $1.3 \mathrm{eV}$ band edge). The inset of Figure 8 shows the current-voltage $(\mathrm{J} / \mathrm{V})$ characteristic of a 6-bilayer CIGSPSS/PEI film deposited on a $200 \mu \mathrm{m} \times 58 \mathrm{~cm}$ channel, in the dark and when illuminating with a $100 \mathrm{~W}$ light source. It is observed that the current density increases by three folds when illuminated by the light source. However, the polymers employed in the LbL deposition process might be susceptible to photodegradation effects, which will be studied in the future, and may require further material design.

3.3. Solar Cell Results. The measured J/V characteristic of the solar cell with $1.0 \mathrm{~cm}^{2}$ active area, when illuminated by a $50 \mathrm{~W}$ light-source, is shown in Figure 9. The considered solar cell structure is shown in the inset of the figure. The open circuit voltage $(\mathrm{V})$, short circuit current density $(\mathrm{J})$, and power density under the illumination were measured to be $0.7 \mathrm{~V}, 0.3 \mathrm{~mA} / \mathrm{cm}^{2}$, and $0.168 \mathrm{~mW} / \mathrm{cm}^{2}$, respectively. The efficiency of the presented solar cell was measured to be $3.5 \%$, which is comparable to the recently reported devices by emerging fabrication techniques such as spray-deposition, but lower than the devices fabricated through coevaporation of the CIGS materials. The LbL assembled CIGS films contain defects that increase carrier recombination. These films are also loosely packed and include polymers that contribute to lower the carrier mobility and thus affect the efficiency of the solar cell. However, it should also be noted that LbL assembly is simple, cost-effective, and highly scalable.

\section{Conclusions}

Fabrication of thin CIGS films using cost-effective, nonvacuum, and highly scalable LbL nanoassembly process has been presented. CIGS nanoparticles were synthesized, dispersed in water, and desired zeta-potential were obtained through $\mathrm{pH}$ regulation (from $+61 \mathrm{mV}$ at $\mathrm{pH} 7$ to $-51 \mathrm{mV}$ at $\mathrm{pH} 10.5$ ) and coating with PSS $(-56.9 \mathrm{mV})$. The LbL film deposition process of the oppositely charged CIGS nanoparticles (CIGS/CIGS), CIGS nanoparticles and PSS (CIGS/PSS), and PSS-coated CIGS nanoparticles and polyethylenimine (CIGS-PSS/PEI) was studied. The J/V and photoelectric characterization results of the films have been presented and discussed. Prototype solar cell devices using the developed films have been fabricated and tested. LbL deposited CIGS films are applicable in cost-effective solar manufacturing.

\section{Acknowledgments}

Acknowledgments are due to the Integrated Nanosystems Development Institute (INDI) for providing the support and facility for this research. The authors are thankful to Jason Cambridge, Mark Canner, Parvin Ghane, Robert Vittoe, and Dr. Daniel Minner for their laboratory assistance.

\section{References}

[1] M. Agarwal, S. Shrestha, P. Ghane, and K. Varahramyan, "Layer-by-Layer nanoassembly of CIS nanoparticles," in Proceedings of the ASME International Manufacturing Science and Engineering Conference, vol. 2, pp. 415-418, Erie, Pa, USA, 2010.

[2] F. N. Crespilho, V. Zucolotto, O. N. Oliveria Jr., and F. C. Nart, "Electrochemistry of layer-by-layer films: a review," International Journal of Electrochemical Science, vol. 1, pp. 194-214, 2006.

[3] I. Repins, M. A. Contreras, B. Egaas et al., "19.9\%-efficient $\mathrm{ZnO} / \mathrm{CdS} / \mathrm{CuInGaSe} \mathrm{C}_{2}$ solar cell with $81.2 \%$ fill factor," Progress in Photovoltaics: Research and Applications, vol. 16, no. 3, pp. 235-239, 2008.

[4] M. Powalla, G. Voorwinden, D. Hariskos, P. Jackson, and R. Kniese, "Highly efficient CIS solar cells and modules made by the co-evaporation process," Thin Solid Films, vol. 517, no. 7, pp. 2111-2114, 2009.

[5] V. K. Kapur, A. Bansal, P. Le, and O. I. Asensio, "Nonvacuum processing of $\mathrm{CuIn}_{1-x} \mathrm{Ga}_{x} \mathrm{Se}_{2}$ solar cells on rigid and 
flexible substrates using nanoparticle precursor inks," Thin Solid Films, vol. 431-432, pp. 53-57, 2003.

[6] F. Bensebaa, C. Durand, A. Aouadou et al., "A new green synthesis method of $\mathrm{CuInS}_{2}$ and $\mathrm{CuInSe}_{2}$ nanoparticles and their integration into thin films," Journal of Nanoparticle Research, vol. 12, no. 5, pp. 1897-1903, 2010.

[7] A. R. Uhl, Y. E. Romanyuk, and A. N. Tiwari, "Thin film $\mathrm{Cu}(\mathrm{In}, \mathrm{Ga}) \mathrm{Se}_{2}$ solar cells processed from solution pastes with polymethyl methacrylate binder," Thin Solid Films, vol. 519, no. 21, pp. 7259-7263, 2011.

[8] K. Varahramyan and Y. Lvov, "Nanomanufacturing by layerby-layer assembly-from nanoscale coating to device applications," Journal of Nanoengineering and Nanosystems, vol. 220, no. 1, pp. 29-37, 2006.

[9] M. G. Panthani, V. Akhavan, B. Goodfellow et al., "Synthesis of $\mathrm{CuInS}_{2}, \mathrm{CuInSe}_{2}$, and $\mathrm{Cu}\left(\mathrm{In}_{x} \mathrm{Ga}_{1-x}\right) \mathrm{Se}_{2}$ (CIGS) nanocrystal "inks" for printable photovoltaics," Journal of the American Chemical Society, vol. 130, no. 49, pp. 16770-16777, 2008.

[10] A. de Kergommeaux, A. Fiore, N. Bruyant et al., "Synthesis of colloidal CuInSe $\mathrm{C}_{2}$ nanocrystals films for photovoltaic applications," Solar Energy Materials and Solar Cells, vol. 95, supplement 1, pp. S39-S43, 2011.

[11] V. A. Akhavan, M. G. Panthani, B. W. Goodfellow, D. K. Reid, and B. A. Korgel, "Thickness-limited performance of CuInSe nanocrystal photovoltaic devices," Optics Express, vol. 18, no. S3, pp. A411-A420, 2010.

[12] Y. Lvov, K. Ariga, I. Ichinose, and T. Kunitake, "Assembly of multicomponent protein films by means of electrostatic layerby-layer adsorption," Journal of the American Chemical Society, vol. 117, no. 22, pp. 6117-6123, 1995. 

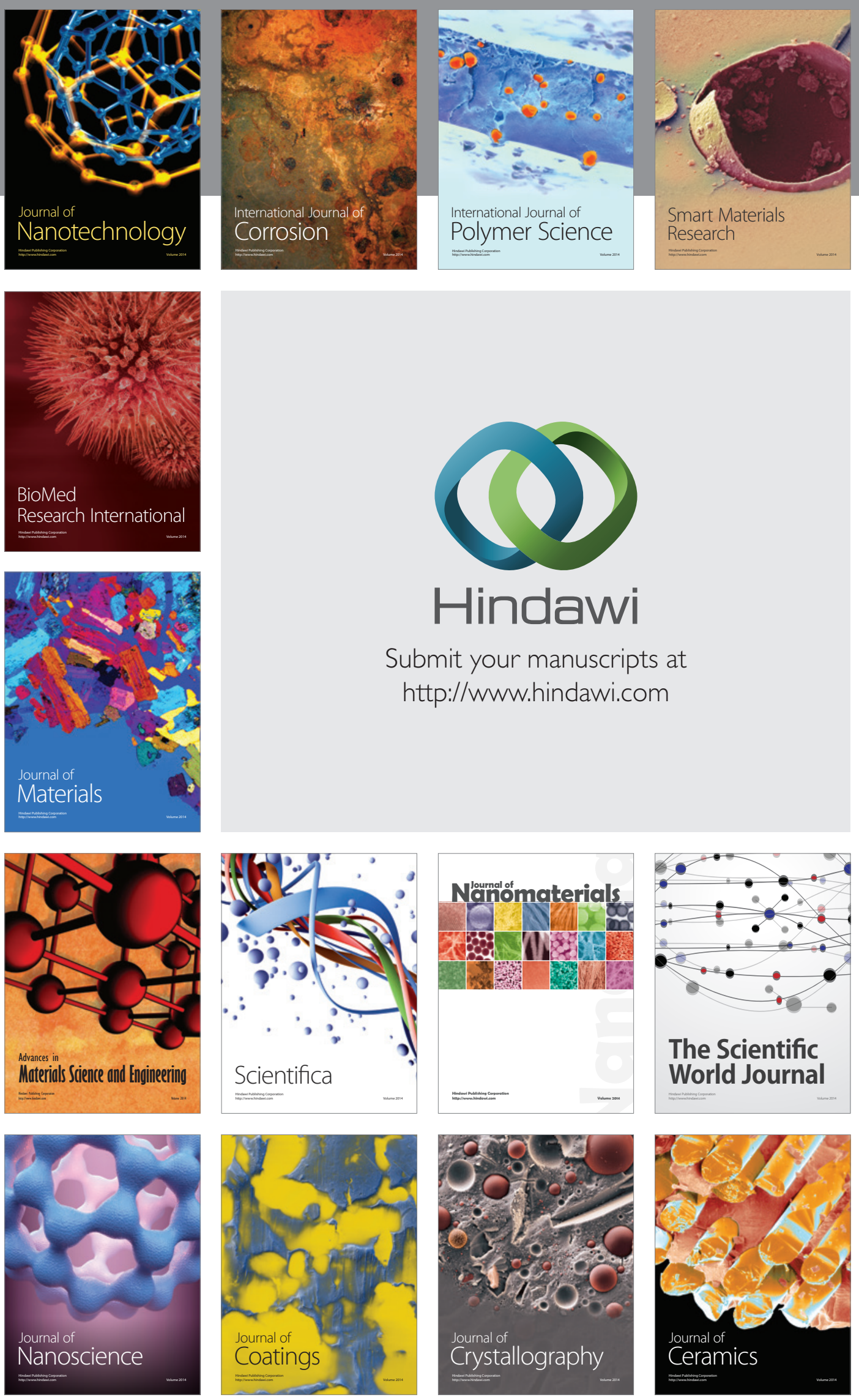

The Scientific World Journal

Submit your manuscripts at

http://www.hindawi.com

\section{World Journal}

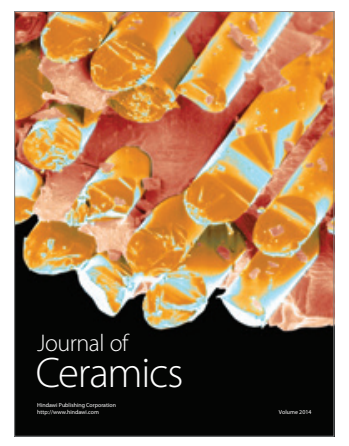

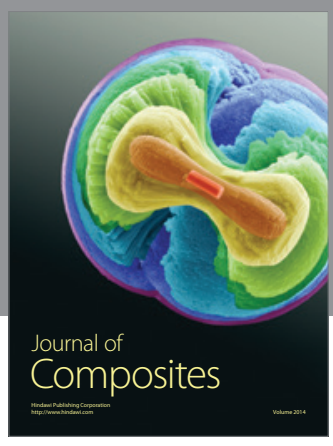
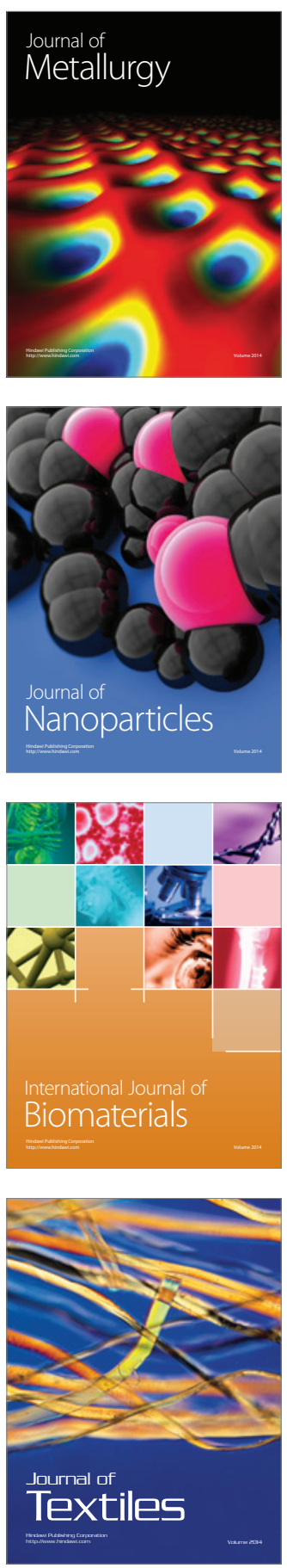